\title{
Using neutron tomography to examine guitar strings
}

Alison Jeanine Edwards ${ }^{1}$, Ulf Garbe ${ }^{1}$, Filomena Salvemini ${ }^{1}$, Ciaran F Edwards-McKeown ${ }^{2}$, John Close ${ }^{3}$, Peter Tregear ${ }^{4}$

${ }^{1}$ Australian Centre For Neutron Scattering, Australian Nuclear Science And Technol, Lucas Heights, Australia, ${ }^{2}$ Arthur St, Marrickville, Australia, ${ }^{3}$ Department of Quantum Science, Physics Education Centre, Australian National University, A.C.T., Australia, ${ }^{4}$ Department of Music, Royal Holloway University of London, Egham,Surrey, United Kingdom E-mail: Alisonedwar@gmail.com

The application of the DINGO neutron imaging facility[1] at ANSTO has opened up the possibility of examining many items of interest across a broad range of cultural pursuits. In the commissioning phase of the instrument build, a project was proposed as part of an undergraduate project in the Australian National University, Department of Physics, to examine as manufactured, and as used guitar strings to probe whether any differences between the two classes of objects could be observed. Guitarists have long asserted the there is a phenomenon of "deadening" which practitioners can hear in the sound produced from strings which have reached the end of their useful lifespan and we were curious to probe whether any observable differences could be discerned.

In undertaking the experiment in the commissioning phase, we were able to exploit the nature of the sample to assist in validation of the procedures employed to create the tomographic reconstructions from the 2-D radiographic images which are recorded across a succession of rotations. We chose to place the strings around a cylindrical support with air in between which allowed a straightforward visual verification of the reconstruction compared directly to the object examined - the approximately linear nature of the components was also useful in this regard (see figure at left below).

The initial examination was undertaken at a resolution of 25 microns and yielded images which gave useful tomographic information in addition to the validation of the procedures being implemented. The metal wound stings were readily identified with strong contrast between interior and exterior materials - in line with expectations. Intriguingly, within the used Nylon strings, apparent variations in contrast were observed (see figure at right below) which are not seen in the new strings. Interesting contrast variations in the metallic windings were also noted in both used and unused strings. At this stage no conclusions have been drawn about whether these variations signify real effects and it is hoped that higher resolution studies may, in the future, provide further insight into the nature of the wear in these and perhaps other instrument strings. It is interesting to note that the predictions by some proposal referees that it would be futile to undertake studies on material including Nylon elements proved to be incorrect.

[1]Garbe, U; Randall, T; Hughes, C, (2011) Nucl. Instrum. Methods Phys. Res., Sect. A 651(1), 42-46

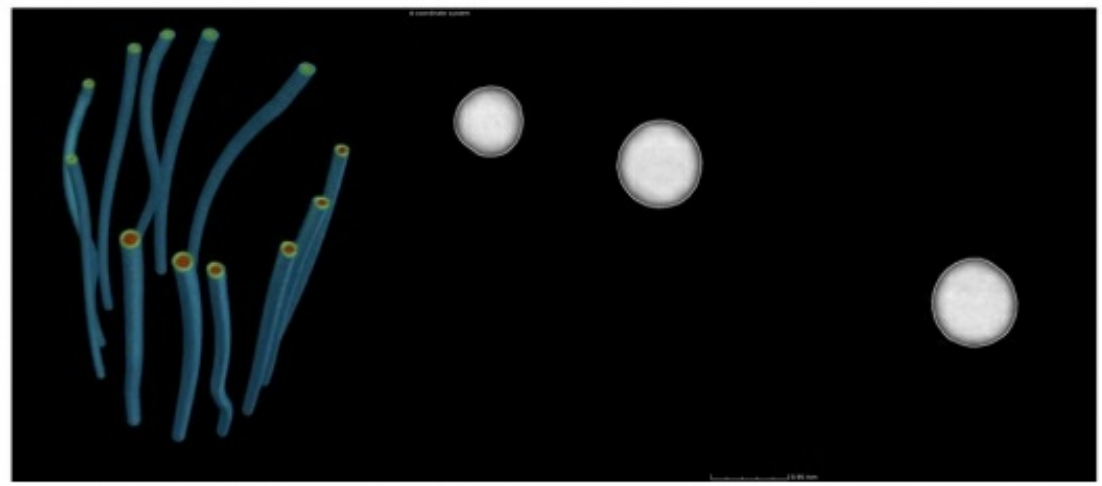

Keywords: neutron, tomography, imaging 